\title{
Annotations of Recurrent Structural Variant Events in Pan-cancer Whole Genome Data for Precision Medicine
}

\section{Takahiko Koyama}

IBM Research - Thomas J. Watson Research Center

Takanori Hasegawa

University of Tokyo

Rika Kasajima

University of Tokyo

Eigo Shimizu

University of Tokyo

Reitaro Tokumasu

IBM Research - Tokyo

Kazuaki Yokoyama

University of Tokyo

Kiyoshi Yamaguchi

University of Tokyo

Arinobu Tojo

University of Tokyo

Yoichi Furukawa

University of Tokyo

Dilhan Weeraratne

IBM Watson Health

Van Willis

IBM Watson Health

Jane Snowdon

IBM Watson Health

Hidewaki Nakagawa

RIKEN

Satoru Miyano

University of Tokyo

\section{Laxmi Parida}

IBM Research - Thomas J. Watson Research Center

Rui Yamaguchi 
Aichi Cancer Center

Seiya Imoto ( $\sim$ imoto@ims.u-tokyo.ac.jp )

Division of Health Medical Data Science, Health Intelligence Center, The Institute of Medical Science, The University of Tokyo, Tokyo, Japan

\section{Research Article}

Keywords: single nucleotide variation (SNV), copy number variation (CNV), PanCancer Analysis of Whole Genomes (PCAWG)

Posted Date: January 4th, 2021

DOI: https://doi.org/10.21203/rs.3.rs-136297/v1

License: (c) (i) This work is licensed under a Creative Commons Attribution 4.0 International License. Read Full License 


\section{Annotations of Recurrent Structural Variant Events in Pan-Cancer Whole Genome Data for Precision Medicine}

Takahiko Koyama ${ }^{1,12}$, Takanori Hasegawa ${ }^{2,12}$, Rika Kasajima², Eigo Shimizu ${ }^{3}$ Reitaro Tokumasu ${ }^{4}$, Kazuaki Yokoyama ${ }^{5,6}$, Kiyoshi Yamaguchi ${ }^{7}$, Arinobu Tojo ${ }^{5,6}$, Yoichi Furukawa ${ }^{7}$, Dilhan Weeraratne ${ }^{8}$, Van Willis ${ }^{8}$, Jane Snowdon ${ }^{8}$, Hidewaki Nakagawa ${ }^{9}$, Satoru Miyano ${ }^{3}$, Laxmi Parida ${ }^{1}$, Rui Yamaguchi ${ }^{3,10,}$ ${ }^{11}$, Seiya Imoto ${ }^{2}$

${ }^{1}$ Computational Genomics, IBM T. J. Watson Research Center, Yorktown Heights, NY, U.S.A.

${ }^{2}$ Division of Health Medical Data Science, Health Intelligence Center, The Institute of Medical Science, The University of Tokyo, Tokyo, Japan

${ }^{3}$ Laboratory of DNA Information Analysis, Human Genome Center, The Institute of Medical Science, The University of Tokyo, Tokyo, Japan

${ }^{4}$ IBM Research- Tokyo, Tokyo, Japan

${ }^{5}$ Division of Molecular Therapy, Advanced Clinical Research Center, The Institute of Medical Science, The University of Tokyo, Tokyo, Japan

${ }^{6}$ Department of Hematology/Oncology, Research Hospital, The Institute of Medical Science, The University of Tokyo, Tokyo, Japan

${ }^{7}$ Division of Clinical Genome Research, Advance Clinical Research Center, The Institute of Medical Science, The University of Tokyo, Tokyo, Japan

${ }^{8}$ Center for Artificial Intelligence, Research and Evaluation, IBM Watson Health, Cambridge, MA, U.S.A.

${ }^{9}$ Laboratory for Cancer Genomics, RIKEN Center for Integrative Medical Sciences, Kanagawa, Japan

${ }^{10}$ Division of Cancer Systems Biology, Aichi Cancer Center Research Institute, Aichi, Japan

${ }^{11}$ Deparment of Cancer Informatics , Nagoya University Graduate School of Medicine, Aichi, Japan

${ }^{12}$ These authors contributed equally to this work. 


\section{Abstract}

In personalized cancer genomic medicine, characterizing a patient's molecular profile based on comprehensive information is important for maximizing treatment benefits. However, current cancer genome analysis is centered on single nucleotide variation (SNV), gene expression, and copy number variation (CNV) but places little emphasis on structural variations (SV) beside fusions. To date, investigation of SVs has been limited because SV analysis entails a cumbersome annotation process. This study describes the design, development, and implementation of an annotation tool for SV, termed SVAnnotator. Detailed annotation was performed on the results of SV detection of 2,781 whole genome samples from the ICGC/TCGA PanCancer Analysis of Whole Genomes (PCAWG) with identifications of fusion, exon skipping, gene disruption, and tandem duplication SVs. These annotations of SVs will facilitate understanding of molecular events and further enhance utilities of precision medicine in stratifications, pathogenicity assessments and drug responses. Frequent novel SV events in MACROD2, FHIT, WWOX and CCSER1 were observed across many cancers. Importantly, SV events were frequently identified in well-established tumor suppressor genes including RB1, NF1, PTEN and TP53. As such, it is plausible that potential therapeutic opportunities are overlooked when SV analysis is not appropriately performed. Given the frequency of SVs detected in our study, SV analysis with detailed annotation should be a routine part of comprehensive precision medicine analysis, and further studies are warranted to enhance clinical benefits as well as our understanding of uncharacterized SV events. 


\section{Introduction}

Structural variations (SV) include deletions (DEL), duplications (DUP), inversions (INV), and translocations (TRA) with lengths typically 1,000 base-pairs (bp) or larger. They are a natural and key component of human variation ${ }^{1}$ and affect a larger portion of the human genome than single nucleotide variants $(\mathrm{SNV})^{2}$. SV events cause not only copy number changes in the genes in a region but also gene disruptions, exon skipping, fusions, dysfunctional fusions, and internal tandem duplications (ITD).

SV events are quite common in cancer due to the inherent chromosomal instability associated with the disease ${ }^{3-8}$. In some cases, such as chromothripsis ${ }^{9-11}$, chromoplexy $^{12}$, and other complex SVs ${ }^{13}$, a tumor may present with multiple SVs acquired as a single event resulting in complex genome rearrangements and genome instability.

In current cancer genomic analysis, copy number variations (CNV) and fusions are typically captured with traditional analytic methodologies; however, subtler events such as gene disruptions, exon skipping, and ITD are often neglected due to the laboriousness of current annotation methodologies for detected SVs. For instance, a typical CNV analysis will likely fail to find partial loss of a gene (defined as disruption in this study) and is also more likely to miss exon skipping and ITD events. It is known, however, that genes which are not prone to point mutations can be altered in SV-induced gene disruption and exon skipping. Common fragile sites (CFS) are an example of this phenomenon ${ }^{14}$. Besides fusions, the current knowledge of SV events in cancer has been primarily limited to EGFRvIII in glioblastoma multiforme (GBM), MET exon 14 skipping in lung adenocarcinoma, AR-V3 and AR-V7 in prostate cancer, and FLT3 ITD in acute myeloid leukemia.

No comprehensive list of recurrent SV events in cancer currently exists, partly due to the complexity of cancer genomes and variety of signals required for the comprehensive detection of SVs ${ }^{15-17}$. The generation of billions of short reads from whole genome sequencing presents challenges to develop algorithms and workflows to identify chromosomal break points ${ }^{18}$; conversely, long read sequencing 
technologies have been developed, but their increased cost and high error rate is not conducive to accurate detection of SV events ${ }^{19,20}$. Recent technological advances in sequencing and algorithmic detection of SVs, however, provide new opportunities for assessing the role of SVs in cancer ${ }^{18,21-24}$. The purpose of this study was to design, develop, and implement a novel SV annotation tool, SVAnnotator, to discover recurrent SV events using International Cancer Genome Consortium (ICGC)/The Cancer Genome Atlas (TCGA) PanCancer Analysis of Whole Genomes (PCAWG) data in multiple cancer types. Identification of clinically targetable SV events will extend the current spectrum of actionable alterations beyond SNVs, CNVs, and fusions and will provide additional targeted treatment options at the point of care.

\section{Results}

Analysis of de-identified 2,781 samples of 31 cancer types from the PCAWG project with the novel SV annotation tool, SVAnnotator, annotated total 200,772 SV events and classified into seven SV types shown in Table 1. The most frequent SVs detected were gene disruptions, followed by dysfunctional fusions, fusions, exon skipping, ITDs, deletions, and deletion-insertion. Detailed results of SV annotations for each gene are provided in Table S1.

Table 2 lists the top recurrent fusion events identified by SVAnnotator with their frequency by cancer type. We compared the frequency of our most commonly observed fusion event, TMPRSS2-ERG in prostate cancer, with the frequency of the same fusion event in the COSMIC database ${ }^{25}$. We identified 124 TMPRSS2-ERG fusions in 248 ICGC samples (44\%) compared with a $38 \%$ frequency $(2303 / 6145)$ in the COSMIC database. Additionally, we identified KIAA1549-BRAF fusion in 64\% (57/89) of pilocytic astrocytoma cases which is consistent with a previously published study where this fusion was detected in $75 \%(24 / 32)$ of cases analyzed ${ }^{26}$. The complete list of fusions obtained from our analysis can be found in Table S2.

Notably, gene disruptions in well-established cancer genes, including PTEN (68), were among the 49 genes most commonly affected by structural variant events in our analysis (Figure 1). Other cancer 
census genes ${ }^{27}$ such as CDK12 (38), RAD51B (37), RB1 (33), TP53 (31), NF1 (26), SMAD4 (23), ARID1A (21), and CDKN2A (20) were also frequently disrupted (Figure 2). Table S3 shows the complete list of dysfunctional fusions found in our analysis.

Exon skipping events were detected in a wide set of genes that have been previously associated with cancer including FHIT (235), MACROD2 (196), WWOX (147), LRP1B (139), PTPRD (122), NAALADL2 (114), CCSER1 (112), PDE4D (108), PARK2 (97), IMMP2L (71), CSMD1 (69), LSAMP (62), GMDS (61), PARD3B (56), RBFOX1 (55), ANKS1B (50), DLG2 (46), AUTS2 (45), and CNTNAP2 (45). Recurrent exon skipping genes in the cancer census list included FOXP1 (36), RUNX1 (32), PTEN (21), RB1 (21), EGFR (19), ARID1B (18), RAD51B (17), and NF1 (16). More details of which exons are skipped for each gene and their associated transcripts can be found in Table S4.

Although the prevalence of ITD events was less than that of exon skipping events, we identified recurrent hitherto unreported ITDs in LRP1B (29), RAD51B (29), NF1 (21), FHIT (18), and RB1 (16). Our evidence underscores the existence of cancer-associated ITD events beyond the well-characterized FLT3-ITD.

Dysfunctional fusions do not result in cohesive mRNAs and thus do not engender stable protein products; however, what's often overlooked is that these fusions lead to the loss of at least one copy of the genes involved leading to loss of heterozygosity. In this study we uncovered two novel dysfunctional fusions in ATAD1-PTEN (12) and PTEN-RNLS (13), rendering PTEN haploinsufficient.

Table 3 lists the most frequent SVs identified for each cancer type. The disease-specific events were selected using chi-square analysis with a threshold of $p$-value $<1.0 \mathrm{e}-25$ (Table S5). Notable findings included 14 GBM cases (35\%) with exon skipping events leading to constitutively active EGFRvIII and other oncogenic isoforms; 35 (11\%) liver cancer and 15 (31\%) lung squamous cell carcinoma cases with LRP1B exon skipping; 24 colorectal cancer cases (40\%) with RBFOX1 exon skipping; over $20 \%$ of bone osteosarcoma cases with DLG2, CSAMP or CNTNAP2 exon skipping; both 5 prime and 3 prime 
TENM4 fusions in melanoma; and 70 cases (80\%) of esophageal adenocarcinoma with FHIT exon skipping located in the FRA3B locus.

\section{Discussion}

While advances in NGS technology have focused on SNVs, CNVs, and fusions as primary oncogenic drivers, structural variants have emerged as an under-appreciated yet integral component of the cancer genome. Limitations in analysis methodologies and availability of whole genome sequencing data have precluded the development of a comprehensive database of SVs in cancer. However, in this work, SVAnnotator was able to identify SV events spanning the entire genome. These included SVs in clinically actionable cancer-associated genes, currently non-targetable cancer-associated genes, and genes not yet associated with cancer but representing potentially novel targets for future investigation.

As expected, many of these top recurrent events were identified at CFS, including FRA3B, FRA2F, and FRA16D loci. Examples from our study include FHIT (localized within FRA3B) which positively regulates antigen presentation ${ }^{28}$; thus, loss of FHIT might negatively affect immune checkpoint inhibitor response $^{29}$. On the contrary, loss of LRP1B (FRA2F) might be associated with positive immune checkpoint inhibitor response ${ }^{30}$. Recently, MACROD2 (FRA20B) is reported to be related to chromosomal instability (CIN) in colorectal cancer $^{31}$, and is mutually exclusive with microsatellite instability (MSI) which is known to respond to immune checkpoint inhibitors ${ }^{32}$. WWOX (FRA16D) has been reported to be responsible for metastasis in triple negative breast cancer ${ }^{33}$. Notably, genes like PTPRD, EYS, TMPRSS2, and ERG do not belong to the currently known CFS. The aforementioned four CFS loci are associated with particular cancer types; however, SV events in PTPRD and EYS are more ubiquitous across many cancer types. Also, our work reiterates the importance of whole genome sequencing to capture these non-CFS loci events.

Detection of SVs in well-established cancer genes underscores the fact that clinically actionable alterations may be missed using traditional genomic screening approaches. Most notable in this class 
of findings was the detection of SV events in NF1, RB1, CDKN2A, SMAD4, TP53, ARID1A/B and PTEN. NF1 is a known tumor suppressor gene which suppresses the MAPK pathway, and tandem duplication events of NF1 were specifically observed in ovarian cancer in our data set. NF1 loss of function mutations are detected in only $2.5 \%$ of the cases in the TCGA ovarian cancer dataset ${ }^{34}$. However, we detected NF1 tandem duplication in 13\% (15/112) of ovarian adenocarcinoma patients. As NF1 inactivation affects the mTOR pathway, disruption of NF1 through SV events represents an additional therapeutic opportunity to use inhibitors targeting the mTOR pathway. RB1 repeats were observed in many cancer types including breast, ovarian, pancreatic, and osteosarcoma. This study identified $4 \%$ (8/195) of breast cancer and 4.5\% (5/112) of ovarian cancer samples had RB1 repeats; loss of function mutations in RB1 have been reported to confer resistance to CDK inhibitors ${ }^{35}$. As such, SV events in RB1 should also be considered when CDK inhibitor treatment is deliberated. CDKN2A, a well-known tumor suppressor gene, shows many disruptions in liver cancer. CDKN2A gene disruptions were identified in 3\% (7/238) of ICGC pancreatic adenocarcinoma cases. Loss of CDKN2A dysregulates the cell cycle and may represent a therapeutic opportunity with CDK inhibitors. SMAD4 copy loss or inactivation was reported in $38 \%$ of pancreatic cancer cases in the TCGA study and is indicative of poor prognosis in pancreatic cancer ${ }^{36,37}$. We detected SMAD4 gene disruptions which would have been overlooked by gene panels and annotation services in $5 \%(12 / 238)$ of pancreatic tumors. TP53 disruptions were observed in $11 \%$ (4/37) of osteosarcoma and $7 \%$ (21/238) of prostate adenocarcinoma samples. While mutations causing loss of function are common in TP53 and PTEN, SVAnnotator detected gene disruptions and dysfunctional fusions which conventional NGS workflows would not have identified. PTEN loss impacts the PI3K pathway and may indicate a targeted therapy opportunity. CDK12 disruptions were mainly found in breast 7\% (13/195), uterus $10 \%(5 / 49)$ and stomach 5\% (4/76) cancers. It is reported that loss of CDK12 confers sensitivity to PARP1 inhibition ${ }^{38}$ and checkpoint inhibitors ${ }^{39}$.

Our study also identified SV events in many genes whose association to cancer is emerging. As such, these findings may not currently affect clinical decisions but present paths for future investigation. 
Key examples (with references to their known associations with cancer) include: LRP $1 \mathrm{~B}^{40}$, MACROD2 $2^{31,41}, \mathrm{RUNX}^{8}, \mathrm{FHIT}^{42}, \mathrm{SPREAD} 1^{43}, \mathrm{CCSER}^{44}, \mathrm{PTPRD}^{45}, \mathrm{DLG}^{46}, \mathrm{SMYD}^{47}$, and $\mathrm{MSI}{ }^{48}$. SV events in genes that hitherto have not been widely associated with cancer were also frequently identified in our work, particularly novel fusions. These include PDE4D, NAALADL2, WWOX, EYS, IMMP2L, ANKS1B, RP11-145E5.5, RAD51B, and ZNF521 among others. High frequency of SV events in these genes warrant further investigation as to their role in cancer and may indicate opportunities for the development of novel therapeutics.

It is difficult to identify break points at single bp resolution and the results of various SV detection tools do not align with one another. This resolution issue and the capricious emergence of pseudo acceptors and donors make in-frame and out-of-frame prediction of resulting gene products even harder. Similarly, the ramifications of some SVs are not easily predicted and multiple complex SV event in a locus cannot be annotated by the SVAnnotator. Also, it should be noted that not all SVs identified, even in tumor suppressor and cancer-related genes, are necessarily associated with cancer risk and development. For example, when oncogenes are amplified, incomplete copies of genes that are snipped can sometimes leave traces observed as gene disruptions, as seen in EGFR disruptions. Dysfunctional fusions as observed in PTEN can lead to lack of heterozygosity in tumor suppressor genes. Thus, SV events, regardless of the type, should be carefully examined using polymerase chain reaction or RNA-seq analysis. Detailed studies should be carried out to confirm the role of SV events in cancer development and pathogenesis.

In summary, the SVAnnotator tool was able to identify various genes that are frequently subjected to SV events in cancer patients. These findings included SV events in well-established cancer genes that are clinically actionable as well as genes with limited association to cancer. Further work is required to elucidate the role of these SVs in tumorigenesis and progression. In conclusion, this study suggests that identification of SVs in cancer can provide additional clinically pertinent insights that may be 
overlooked by conventional genomic analysis workflows and as such should be integrated as a routine practice of comprehensive precision medicine analysis.

\section{Materials and methods}

\section{Samples}

All samples used in this study were derived from the Pan-Cancer Analysis of Whole Genomes (PCAWG) study subset of the ICGC final consensus genome data (2,781 total samples across 31 cancer types). We used the results of somatic structural variant detection analysis performed in the PCAWG project. For $67.3 \%$ of samples, tumors were freshly frozen solid samples whereas controls were blood samples. In contrast, for $20.2 \%$ of samples, controls were derived from tumor-adjacent normal tissues or other non-blood tissues, particularly in hematological cancers ${ }^{49} .93 .8 \%$ of the tumor samples came from treatment-free primary cancers, but $6.2 \%$ originated from donors with multiple samples of primary, metastatic, and/or recurrent tumors. Due to large contributions from European, North American, and Australian genomic projects, the continental ancestry distribution of the sample was heavily weighted towards Europeans (77\%) and East Asians $(16 \%)^{49,50}$.

\section{Sequencing}

Sequencing was performed by each data provider of the PCAWG project and Illumina whole-genome paired-end sequencing, which reads lengths of $100-150 \mathrm{bp}$ from tumor and normal samples was used. The mean whole genome sequencing coverage in this set was 30 reads per bp for control samples, whereas tumors had a bimodal coverage distribution with maximums at 38 and 60 reads per $\mathrm{bp}^{49,50}$. The BWA-MEM v0.7.17 algorithm ${ }^{51}$ was used to align each tumor and normal sample to Human Genome Reference Build GRCh3752, version hs37d5.

\section{Structural variant calling}

In order to generate a consistent call set to be used for cross-tumor type analysis, all samples were analyzed using a uniform set of algorithms for alignment, variant calling, and quality control. The SV 
call set was generated by one of the working groups of the PCAWG study $y^{50,53,54}$. The call set was generated by merging the SV calls from independent calling pipelines, and the merged SV calls were further required to have a consistent copy number change. The data of analysis results are publicity available at ICGC Data Portal, https://dcc.icgc.org/releases/PCAWG.

\section{Structural variant annotation; SVAnnotator}

We developed an annotation tool named SVAnnotator to automate the cumbersome task of transforming structural variant data into human comprehensible events such as fusions, exon skipping, ITD, and gene disruptions. SVAnnotator takes 2 break points' coordinates, their connection orientations $\left(5^{\prime}-5^{\prime}, 5^{\prime}-3^{\prime}, 3^{\prime}-5^{\prime}, 3^{\prime}-3^{\prime}\right)$, and types (INV, DEL, DUP, TRA) as inputs; SVAnnotator uses tab separated text files with coordinates, strand orientations, and types of event as inputs. BEDPE files generated by an SV caller can be easily transformed into tab separated text files by a simple script or a spreadsheet. Variant Call Format (VCF) and BND format can be transformed by Bioconductor ${ }^{55}$ or other freely available tools. The headers must start with ' $\#$ ' if any. Most SV callers provide ranges rather than chromosomal position for break points; however, SVAnnotator cannot accept ranges so users need to pick a position. Also, it is important to remove false positives which originate from alignment issues in low complexity regions or other causes ${ }^{56}$. The overall program flow of SVAnnotator is illustrated in Figure S1. First, the program checks the location of break points using gene definitions as defined in a General Transfer Format (GTF) file. If both break points fall in the same transcript, SVAnnotator classifies it into subtypes (1-9) as shown in Figure S2, considering break point orientations, gene orientation, and connection directions before translation into an event like exon skipping and ITD. SV events with a transcript and an intergenic locus are considered gene disruptions. SV events with a transcript and an intergenic locus are considered gene disruptions. SVAnnotator defines a disruption as either end of a transcript being connected to an intergenic region and the gene effectively considered as a copy loss; whereas a deletion is defined as an event in which internal transcript parts are missing. SV events where both break points fall in two distinct transcripts are 
classified as either fusions identified by $5 / 3$ prime gene or dysfunctional fusions, after considering strand directions and connection orientations, as illustrated in Figure S3. SVAnnotator identifies loss of an acceptor or a donor side of an exon as exon skipping. However, these losses can be indicative of non-exon skipping events ${ }^{57}$; it is strongly encouraged that users check actual transcripts to confirm.

\section{Identifying tumor-type-specific SV events}

To identify SV events that are specifically enriched in some tumor types, a Chi-Square goodness-of-fit test was employed for each SV event that was characterized by an affected gene name and an SV type. The test evaluates how a set of observed counts of a specific SV event across multiple tumor types differs from that of expected ones, which are proportional to the sample sizes of the tumor types.

We restricted our analysis to tumor types with greater than 20 samples and focused on the following 27 cancer subtypes: Biliary.AdenoCA (34), Bladder.TCC (23), Bone.Osteosarc (37), Breast.AdenoCA (195), CNS.GBM (40), CNS.Medullo (147), CNS.PiloAstro (89), ColoRect.AdenoCA (60), Eso.AdenoCA (88), Head.SCC (57), Kidney.ChRCC (45), Kidney.RCC (144), Liver.HCC (323), Lung.AdenoCA (37), Lung.SCC (48), Lymph.BNHL (107), Lymph.CLL (96), Myeloid.AML (29), Myeloid.MPN (58), Ovary.AdenoCA (112), Panc.AdenoCA (238), Panc.Endocrine (85), Prost.AdenoCA (284), Skin.Melanoma (106), Stomach.AdenoCA (76), Thy.AdenoCA (48), and Uterus.AdenoCA (49), where the number in parentheses after each tumor type is the sample size, and the total number of samples is 2,655 . We removed 126 samples belonging to 11 other cancer types of smaller cohorts from the analysis to avoid statistical skewing for cancer type specific event extraction.

For each SV event that was characterized by an affected gene name and an SV type, the Chi-Square goodness-of-fit test was applied to the observed counts of the SV across the 27 tumor types. The total number of distinct SV events considered was 99,498 . The result, i.e., p-values and the observed counts of SV events, are shown in Table S5. 


\section{Software Availability}

SVAnnotator is available from https://github.ibm.com/ComputationalGenomics/

\section{References}

1 Sudmant, P. H. et al. An integrated map of structural variation in 2,504 human genomes. Nature 526, 75-81, doi:10.1038/nature15394 (2015).

2 Pang, A. W. et al. Towards a comprehensive structural variation map of an individual human genome. Genome Biol 11, R52-R52, doi:10.1186/gb-2010-11-5-r52 (2010).

3 Jacoby, M. A. \& Walter, M. J. Detection of copy number alterations in acute myeloid leukemia and myelodysplastic syndromes. Expert review of molecular diagnostics 12, 253264, doi:10.1586/erm.12.18 (2012).

4 Weischenfeldt, J., Symmons, O., Spitz, F. \& Korbel, J. O. Phenotypic impact of genomic structural variation: insights from and for human disease. Nat Rev Genet 14, 125-138, doi:10.1038/nrg3373 (2013).

5 Yang, L. et al. Diverse mechanisms of somatic structural variations in human cancer genomes. Cell 153, 919-929, doi:10.1016/j.cell.2013.04.010 (2013).

Greer, S. U. et al. Linked read sequencing resolves complex genomic rearrangements in gastric cancer metastases. Genome Med 9, 57-57, doi:10.1186/s13073-017-0447-8 (2017).

7 Rahal, Z., Abdulhai, F., Kadara, H. \& Saab, R. Genomics of adult and pediatric solid tumors. Am J Cancer Res 8, 1356-1386 (2018).

8 Viswanathan, S. R. et al. Structural Alterations Driving Castration-Resistant Prostate Cancer Revealed by Linked-Read Genome Sequencing. Cell 174, 433-447.e419, doi:10.1016/j.cell.2018.05.036 (2018).

9 Stephens, P. J. et al. Massive genomic rearrangement acquired in a single catastrophic event during cancer development. Cell 144, 27-40, doi:10.1016/j.cell.2010.11.055 (2011). 
10 Kloosterman, W. P. et al. Chromothripsis is a common mechanism driving genomic rearrangements in primary and metastatic colorectal cancer. Genome Bio/ 12, R103-R103, doi:10.1186/gb-2011-12-10-r103 (2011).

11 Rode, A., Maass, K. K., Willmund, K. V., Lichter, P. \& Ernst, A. Chromothripsis in cancer cells: An update. Int J Cancer 138, 2322-2333, doi:10.1002/ijc.29888 (2016).

12 Baca, S. C. et al. Punctuated evolution of prostate cancer genomes. Cell 153, 666-677, doi:10.1016/j.cell.2013.03.021 (2013).

$13 \mathrm{Yi}, \mathrm{K}$. \& Ju, Y. S. Patterns and mechanisms of structural variations in human cancer. Exp Mol Med 50, 98-98, doi:10.1038/s12276-018-0112-3 (2018).

14 Ma, K. et al. Common fragile sites: genomic hotspots of DNA damage and carcinogenesis. Int J Mol Sci 13, 11974-11999, doi:10.3390/ijms130911974 (2012).

15 Alkan, C., Coe, B. P. \& Eichler, E. E. Genome structural variation discovery and genotyping. Nature reviews. Genetics 12, 363-376, doi:10.1038/nrg2958 (2011).

16 Collins, R. L. et al. Defining the diverse spectrum of inversions, complex structural variation, and chromothripsis in the morbid human genome. Genome Biol 18, 36, doi:10.1186/s13059017-1158-6 (2017).

17 Negrini, S., Gorgoulis, V. G. \& Halazonetis, T. D. Genomic instability--an evolving hallmark of cancer. Nat Rev Mol Cell Biol 11, 220-228, doi:10.1038/nrm2858 (2010).

18 Liu, B. et al. Structural variation discovery in the cancer genome using next generation sequencing: computational solutions and perspectives. Oncotarget 6, 5477-5489, doi:10.18632/oncotarget.3491 (2015).

19 Ewing, A. \& Semple, C. Breaking point: the genesis and impact of structural variation in tumours. F1000Res 7, doi:10.12688/f1000research.16079.1 (2018).

20 Sedlazeck, F. J., Lee, H., Darby, C. A. \& Schatz, M. C. Piercing the dark matter: bioinformatics of long-range sequencing and mapping. Nat Rev Genet 19, 329-346, doi:10.1038/s41576018-0003-4 (2018). 
21 Elyanow, R., Wu, H.-T. \& Raphael, B. J. Identifying structural variants using linked-read sequencing data. Bioinformatics (Oxford, England) 34, 353-360, doi:10.1093/bioinformatics/btx712 (2017).

22 Marks, P. et al. Resolving the full spectrum of human genome variation using Linked-Reads. Genome Res 29, 635-645, doi:10.1101/gr.234443.118 (2019).

23 Nakagawa, H. \& Fujita, M. Whole genome sequencing analysis for cancer genomics and precision medicine. Cancer Sci 109, 513-522, doi:10.1111/cas.13505 (2018).

24 Tattini, L., D'Aurizio, R. \& Magi, A. Detection of Genomic Structural Variants from NextGeneration Sequencing Data. Front Bioeng Biotechnol 3, 92-92, doi:10.3389/fbioe.2015.00092 (2015).

25 Forbes, S. A. et al. COSMIC: exploring the world's knowledge of somatic mutations in human cancer. Nucleic Acids Res 43, D805-811, doi:10.1093/nar/gku1075 (2015).

26 Faulkner, C. et al. BRAF Fusion Analysis in Pilocytic Astrocytomas: KIAA1549-BRAF 15-9 Fusions Are More Frequent in the Midline Than Within the Cerebellum. J Neuropathol Exp Neurol 74, 867-872, doi:10.1097/NEN.0000000000000226 (2015).

27 Sondka, Z. et al. The COSMIC Cancer Gene Census: describing genetic dysfunction across all human cancers. Nat Rev Cancer 18, 696-705, doi:10.1038/s41568-018-0060-1 (2018).

28 Romero, I. et al. The tumour suppressor Fhit positively regulates MHC class I expression on cancer cells. J Pathol 227, 367-379, doi:10.1002/path.4029 (2012).

29 Romero, I. et al. MHC Intratumoral Heterogeneity May Predict Cancer Progression and Response to Immunotherapy. Front Immuno/ 9, 102, doi:10.3389/fimmu.2018.00102 (2018). 30 Hogan, S. A., Levesque, M. P. \& Cheng, P. F. Melanoma Immunotherapy: Next-Generation Biomarkers. Front Oncol 8, 178, doi:10.3389/fonc.2018.00178 (2018).

31 Sakthianandeswaren, A., Parsons, M. J., Mouradov, D. \& Sieber, O. M. MACROD2 deletions cause impaired PARP1 activity and chromosome instability in colorectal cancer. Oncotarget 9, 33056-33058, doi:10.18632/oncotarget.25887 (2018). 
32 Zaravinos, A. et al. Cytolytic activity correlates with the mutational burden and deregulated expression of immune checkpoints in colorectal cancer. J Exp Clin Cancer Res 38, 364, doi:10.1186/s13046-019-1372-z (2019).

33 Chang, R. et al. Loss of Wwox drives metastasis in triple-negative breast cancer by JAK2/STAT3 axis. Nat Commun 9, 3486, doi:10.1038/s41467-018-05852-8 (2018).

34 Cancer Genome Atlas Research, N. Integrated genomic analyses of ovarian carcinoma. Nature 474, 609-615, doi:10.1038/nature10166 (2011).

35 Condorelli, R. et al. Polyclonal RB1 mutations and acquired resistance to CDK 4/6 inhibitors in patients with metastatic breast cancer. Annals of oncology : official journal of the European Society for Medical Oncology 29, 640-645, doi:10.1093/annonc/mdx784 (2018). Demagny, H. \& De Robertis, E. M. Point mutations in the tumor suppressor Smad4/DPC4 enhance its phosphorylation by GSK3 and reversibly inactivate TGF- $\beta$ signaling. Mol Cell Oncol 3, e1025181-e1025181, doi:10.1080/23723556.2015.1025181 (2015).

37 Shugang, X. et al. Prognostic Value of SMAD4 in Pancreatic Cancer: A Meta-Analysis. Trans/ Oncol 9, 1-7, doi:10.1016/j.tranon.2015.11.007 (2016).

38 Paculova, H. \& Kohoutek, J. The emerging roles of CDK12 in tumorigenesis. Cell Div 12, 7, doi:10.1186/s13008-017-0033-x (2017).

39 Wu, Y. M. et al. Inactivation of CDK12 Delineates a Distinct Immunogenic Class of Advanced Prostate Cancer. Cell 173, 1770-1782 e1714, doi:10.1016/j.cell.2018.04.034 (2018).

40 Liu, C.-X., Musco, S., Lisitsina, N. M., Yaklichkin, S. Y. \& Lisitsyn, N. A. Genomic Organization of a New Candidate Tumor Suppressor Gene, LRP1B. Genomics 69, 271-274, doi:https://doi.org/10.1006/geno.2000.6331 (2000).

41 Sakthianandeswaren, A. et al. MACROD2 Haploinsufficiency Impairs Catalytic Activity of PARP1 and Promotes Chromosome Instability and Growth of Intestinal Tumors. Cancer Discov 8, 988-1005, doi:10.1158/2159-8290.Cd-17-0909 (2018). 
42 Pekarsky, Y., Zanesi, N., Palamarchuk, A., Huebner, K. \& Croce, C. M. FHIT: from gene discovery to cancer treatment and prevention. The Lancet Oncology 3, 748-754, doi:https://doi.org/10.1016/S1470-2045(02)00931-2 (2002).

43 Halaban, R. \& Krauthammer, M. RASopathy Gene Mutations in Melanoma. Journal of Investigative Dermatology 136, 1755-1759, doi:https://doi.org/10.1016/i.jid.2016.05.095 (2016).

44 Patel, K. et al. FAM190A deficiency creates a cell division defect. Am J Pathol 183, 296-303, doi:10.1016/j.ajpath.2013.03.020 (2013).

45 Veeriah, S. et al. The tyrosine phosphatase PTPRD is a tumor suppressor that is frequently inactivated and mutated in glioblastoma and other human cancers. Proc Natl Acad Sci U S A 106, 9435-9440, doi:10.1073/pnas.0900571106 (2009).

46 Shao, Y. W. et al. Cross-species genomics identifies DLG2 as a tumor suppressor in osteosarcoma. Oncogene 38, 291-298, doi:10.1038/s41388-018-0444-4 (2019).

47 Sarris, M. E., Moulos, P., Haroniti, A., Giakountis, A. \& Talianidis, I. Smyd3 Is a Transcriptional Potentiator of Multiple Cancer-Promoting Genes and Required for Liver and Colon Cancer Development. Cancer Cell 29, 354-366, doi:10.1016/j.ccell.2016.01.013 (2016).

48 Kang, M. H. et al. Musashi RNA-binding protein 2 regulates estrogen receptor 1 function in breast cancer. Oncogene 36, 1745-1752, doi:10.1038/onc.2016.327 (2017).

49 Campbell, P. J., Getz, G., Stuart, J. M., Korbel, J. O. \& Stein, L. D. Pan-cancer analysis of whole genomes. bioRxiv, 162784, doi:10.1101/162784 (2017).

50 Rodriguez-Martin, B. et al. Pan-cancer analysis of whole genomes reveals driver rearrangements promoted by LINE-1 retrotransposition in human tumours. bioRxiv, 179705, doi:10.1101/179705 (2018).

51 Li, H. \& Durbin, R. Fast and accurate short read alignment with Burrows-Wheeler transform. Bioinformatics 25, 1754-1760, doi:10.1093/bioinformatics/btp324 (2009). 
52 Church, D. M. et al. Modernizing reference genome assemblies. PLoS Bio/ 9, e1001091, doi:10.1371/journal.pbio.1001091 (2011).

$53 \mathrm{Li}$, Y. et al. Patterns of structural variation in human cancer. bioRxiv, 181339, doi:10.1101/181339 (2017).

54 Wala, J. A. et al. Selective and mechanistic sources of recurrent rearrangements across the cancer genome. bioRxiv, 187609, doi:10.1101/187609 (2017).

55 D. Bates, V. C., M. Dettling, S. Dudoit, B. Ellis, L. Gautier, R., Gentleman, J. G., K. Hornik, T. Hothorn, W. Huber, S. lacus, R., Irizarry, F. L., M. Maechler, A. Rossini, G. Sawitzki, L. Tierney, \& J.Y.H. Yang, J. Z. Bioconductor, <http://www.bioconductor.org/> (2002).

56 Cameron, D. L., Di Stefano, L. \& Papenfuss, A. T. Comprehensive evaluation and characterisation of short read general-purpose structural variant calling software. Nat Commun 10, 3240, doi:10.1038/s41467-019-11146-4 (2019).

57 Ito, K. et al. Identification of pathogenic gene mutations in LMNA and MYBPC3 that alter RNA splicing. Proc Natl Acad Sci U S A 114, 7689-7694, doi:10.1073/pnas.1707741114 (2017).

\section{Acknowledgements}

We would like to thank the PCAWG Technical Working group and The PCAWG Network for providing the whole genome data for this study. Super-computing resources were provided by Human Genome Center, the Institute of Medical Science, the University of Tokyo (http://sc.hgc.jp/shirokane.html).

\section{Author information}

\section{Affiliations}

Computational Genomics, IBM T. J. Watson Research Center, Yorktown Heights, NY, U.S.A. 
Takahiko Koyama, Laxmi Parida

Division of Health Medical Data Science, Health Intelligence Center, The Institute of Medical Science, The University of Tokyo, Tokyo, Japan

Takanori Hasegawa, Seiya Imoto

Division of Health Medical Intelligence, Human Genome Center, The Institute of Medical Science, The University of Tokyo, Tokyo, Japan

Rika Kasajima, Eigo Shimizu, Satoru Miyano, Rui Yamaguchi, Seiya Imoto

IBM Research- Tokyo, Tokyo, Japan

Reitaro Tokumasu

Division of Molecular Therapy, Advanced Clinical Research Center, The Institute of Medical Science, The University of Tokyo, Tokyo, Japan

Kazuaki Yokoyama, Arinobu Tojo

Division of Clinical Genome Research, Advance Clinical Research Center, The Institute of Medical Science, The University of Tokyo, Tokyo, Japan

Kazuaki Yokoyama, Arinobu Tojo

Division of Clinical Genome Research, Advance Clinical Research Center, The Institute of Medical Science, The University of Tokyo, Tokyo, Japan

Kiyoshi Yamaguchi, Yoichi Furukawa

Center for Artificial Intelligence, Research and Evaluation, IBM Watson Health, Cambridge, MA, U.S.A.

Dilhan Weeraratne, Van Willis, Jane Snowdon

Laboratory for Cancer Genomics, RIKEN Center for Integrative Medical Sciences, Kanagawa, Japan

Hidewaki Nakagawa 
Division of Cancer Systems Biology, Aichi Cancer Center Research Institute, Aichi, Japan

Rui Yamaguchi

\section{Contributions}

TK and SI conceived and designed the study. TK and LP created software used in this work. TK, TH, RK, ES, DW, VW, JS, RY and SI conducted the data analysis. RT, KaY, KiY, SM and LP helped with data analysis. TK, TH, RT, KaY, KiY, AT, YF, HN, SM, LP, RY and SI conducted data interpretation. RT, DW, VW, JS and HN helped with manuscript preparation. TK, TH, DW, VW, JS, RY and SI wrote and edited the manuscript.

Corresponding author

Correspondence to Seiya Imoto (imoto@ims.u-tokyo.ac.jp)

\section{Ethics declarations}

\section{Competing Interests}

TK, RT, DW, VW, JS, and LP were all employed by IBM at the time this work was conducted. The

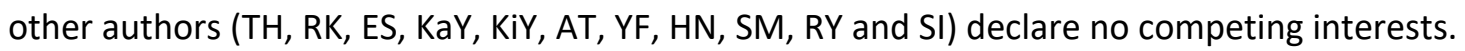




\section{Legends (Tables and Figures)}

Table 1. Number of events by SV type in total and distinct gene and event combinations. SV = structural variant, ITD = internal tandem duplication, $\mathrm{DEL}=$ deletion, $\mathrm{DELINS}=$ deletion-insertion

Table 2. Top recurrent fusion events and their frequency in each cancer type.

Table 3. Most frequent SV events by cancer type. ES = exon skipping, DF=Dysfunctional Fusion, DIS=disruption, $\mathrm{F} 3=$ fusion 3 prime, $\mathrm{F} 5=$ fusion 5 prime, ITD = internal tandem duplication, N/A = not available

Figure 1. Heatmap of the top recurrent SV events. Color represents frequency of the SV for the given cancer type.

Figure 2. Heatmap of the SV events among cancer census genes. Color represents frequency of the SV for the given cancer type. 
Figures

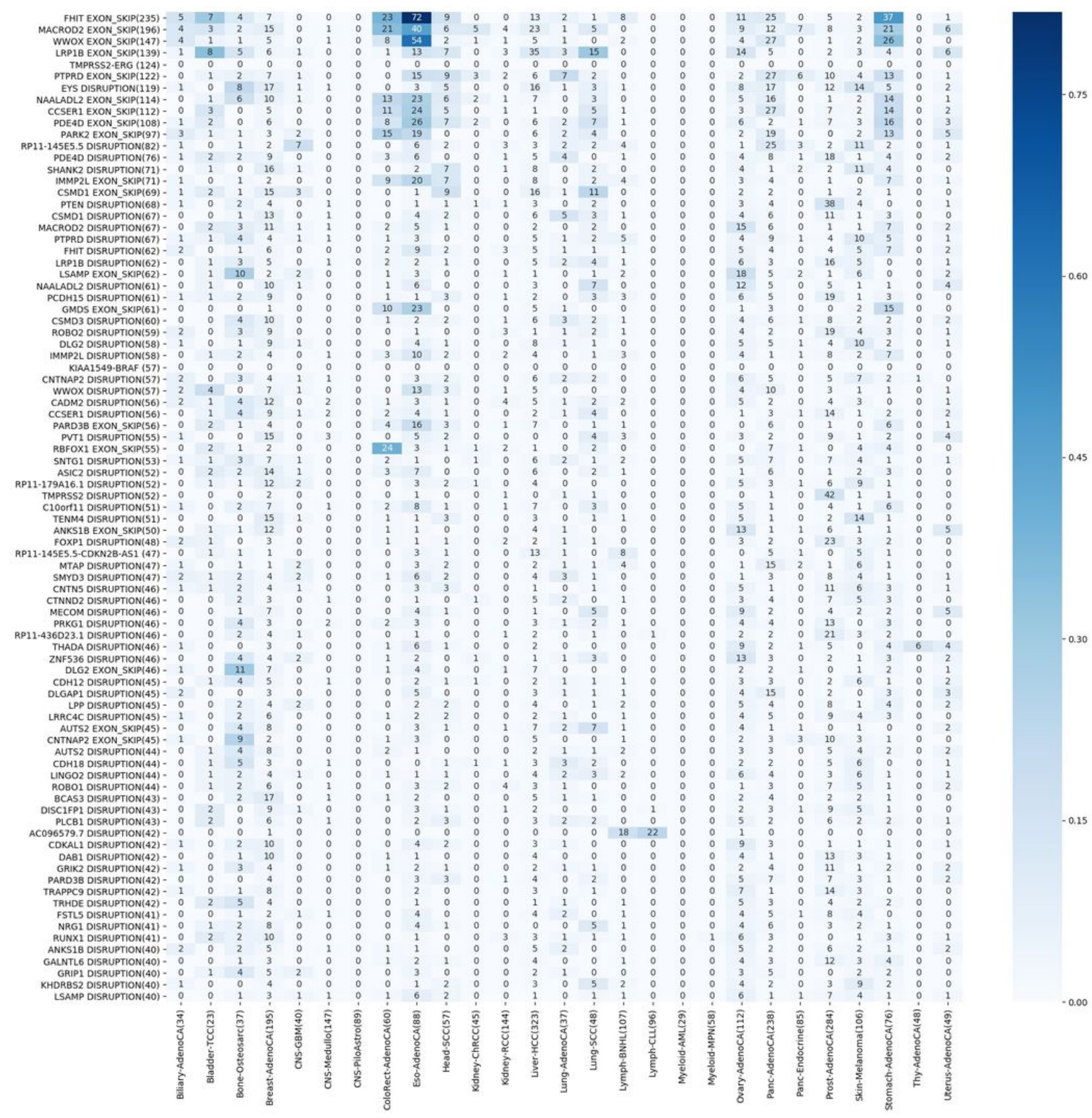

Figure 1

Heatmap of the top recurrent SV events. Color represents frequency of the SV for the given cancer type. 


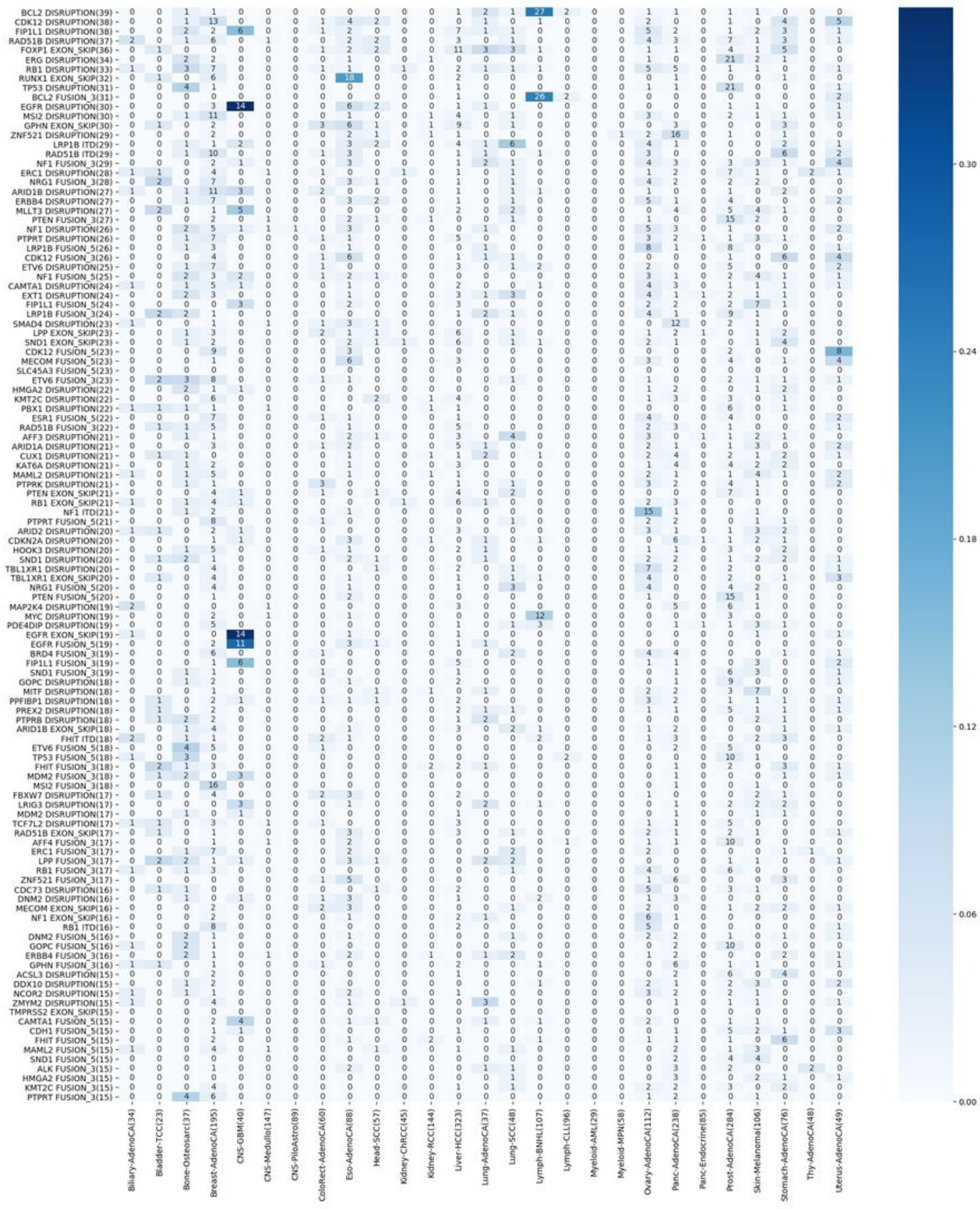

Figure 2

Heatmap of the SV events among cancer census genes. Color represents frequency of the SV for the given cancer type.

\section{Supplementary Files}


This is a list of supplementary files associated with this preprint. Click to download.

- FigureS1svflowchartv1.pdf

- FigureS2svsubtypesdiagramv1.pdf

- FigureS3svfusiondiagramv1.pdf

- SVAnnotatorSupplementaryInformation.pdf

- TableS1.xIsx

- Tables2fusions.xlsx

- TableS3dysfunctionalfusions.xlsx

- TableS4exonskipping.xlsx

- TableS5.xlsx

- Table1.pdf

- Table2.pdf

- Table3.pdf 